\title{
Retroperitoneal Malignant Peripheral Nerve Sheath Tumour without Neurofibromatosis: A Case Report
}

\author{
Veeranathreddy $\mathrm{V}^{\mathrm{b}}$ Jagadish $\mathrm{B}^{\mathrm{a}}$, Puranik $\mathrm{G}^{\mathrm{a}}$,Gopinath $\mathrm{P}^{\mathrm{a}}$,Chidananda $\mathrm{KV}^{\mathrm{a}}$, \\ Ahalya reddy ${ }^{\mathrm{c}}$, Srikanth $\mathrm{CH}^{\mathrm{b}}$, Rahul M Sharma ${ }^{\mathrm{b}}$. \\ A, Professor, b, Post graduate, Department of General Surgery, c, post graduate, Department of Pathology \\ KVG Medical College \&Hospital, Sullia-DK, Karnataka, India.
}

\begin{abstract}
MPNST often arises from a major peripheral nerve. MPNST replaced olden terms like malignant schwannoma, neurofibrosarcoma, neurogenic sarcoma, malignant neurilemmoma .They can occur sporadically or in the context of neurofibromatosis type 1(von Recklinghausen's disease). MPNST is an exception to sarcoma which will not arise from benign precursor. Common sites are major/proximal nerve trunks.

we came across one such patient who presents with mass per abdomen since 2 months, which was insidious in onset, initially $5 \times 5 \mathrm{~cm}$, rapidly progressed to $13 \times 12 \mathrm{~cm}$. On examination mass was retroperitoneal debulking of the tumour was done, Biopsy report suggestive of MPNST.

Nerve sheath tumours have varied clinical presentations, and a systematic approach, with judicious use of imaging, histopathology needed for diagnosis.
\end{abstract}

Keywords: MPNST, Retroperitoneal, Von Recklinghausen's disease.

\section{Introduction}

Peripheral nerve sheath tumours presents as soft tissue neoplasm's and include the spectrum of schwannomas, neurofibromas, neurofibromatosis, and malignant peripheral nerve sheath tumours. The presentation varies from asymptomatic soft tissue swellings to large masses that are recurrent and difficult to excise $^{1}$.

\section{Case report}

A 68 year old male from kodagu admitted with chief complaints of mass per abdomen since 2 months, which was insidious in onset, initially $5 \times 5 \mathrm{~cm}$, rapidly progressed to $13 \times 12 \mathrm{~cm}$, not associated with pain. No h/o jaundice, hemetemesis, melena. No h/o loss of appetite or easy fatigability. No h/o loss of weight, urinary disturbances.

On examination of abdomen, a $15 \times 12 \mathrm{~cm}$ ovoid mass is present in epigastric and umbilical region extending across the midline up to the left lumbar, all borders were well made out, surface is smooth, firm in consistency, non tender, transmitted pulsations felt, does not move with respiration, non ballotable on bimanual palpation, fingers can be insunated below left costal margins, no renal angle fullness/tenderness, no hepatomegaly and splenomegaly. Mass dull to percuss. Auscultation bowel sounds heard.

On admission patient was haemodynamically stable. Routine blood tests are within normal limits.USG abdomen,Well defined homogenously hyperechoic mass lesion in the left upper quadrant of the abdomen displacing the spleen superiorly and the left kidney medially- Retroperitoneal mass lesion. CT abdomen, Well defined poorly circumscribed large heterogeneous retroperitoneal mass lesion with predominant fat component in the entire left quadrants of the abdomen arising from the left posterior pararenal space displacing the viscera as mentioned above infiltrating the left kidney-? Liposarcoma. Biopsy report suggestive of Malignant peripheral nerve sheath tumour.

Exploratory Laparotomy done. Intra operatively tumour is retroperitoneal in left side pushing the descending colon medially. Debulking of the tumour is done. Patient tolerated the surgery well. Post operative period was uneventful. Patient was discharged. Follow up after 15 days with histopathology, sections are densely cellular, tumour is composed of spindle cells arranged in fascicular pattern. The neoplasm display variable pleomorphism with some areas being highly pleomorphic (moderate to severe) with abnormal nuclear contours like buckle, giant and irregular nuclei while other areas appear relatively less pleomorphic (mild to moderate) and quite monotonus in appearance. Areas of necrosis and dense round cell inflammatory infiltrate are seen focally. Mitosis is a little sparse and variable from field to field. Nuclear palisading similar to that of neural neoplasms is observed in some of the sections. The background shows a fine fibrillary network. Suggestive of MPNST. 


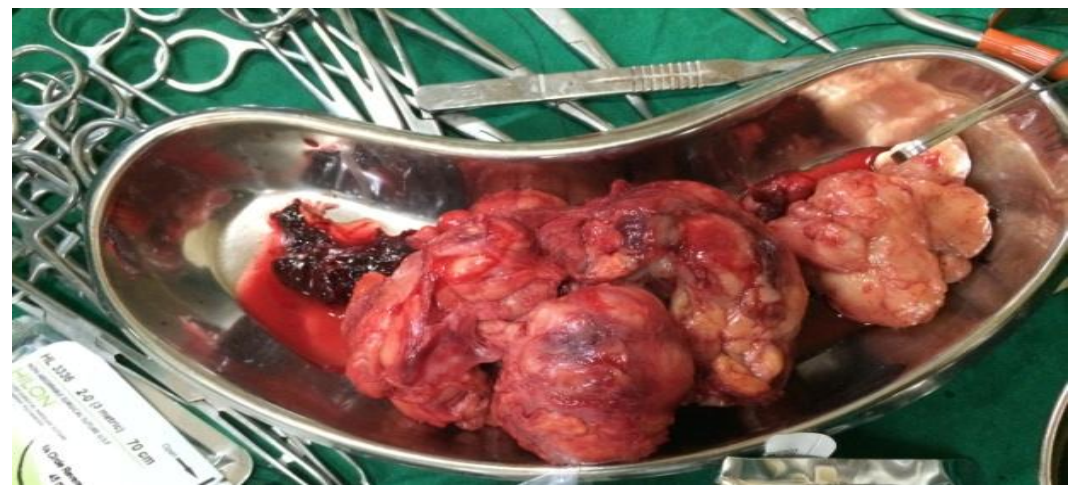

Figure 1 Gross specimen
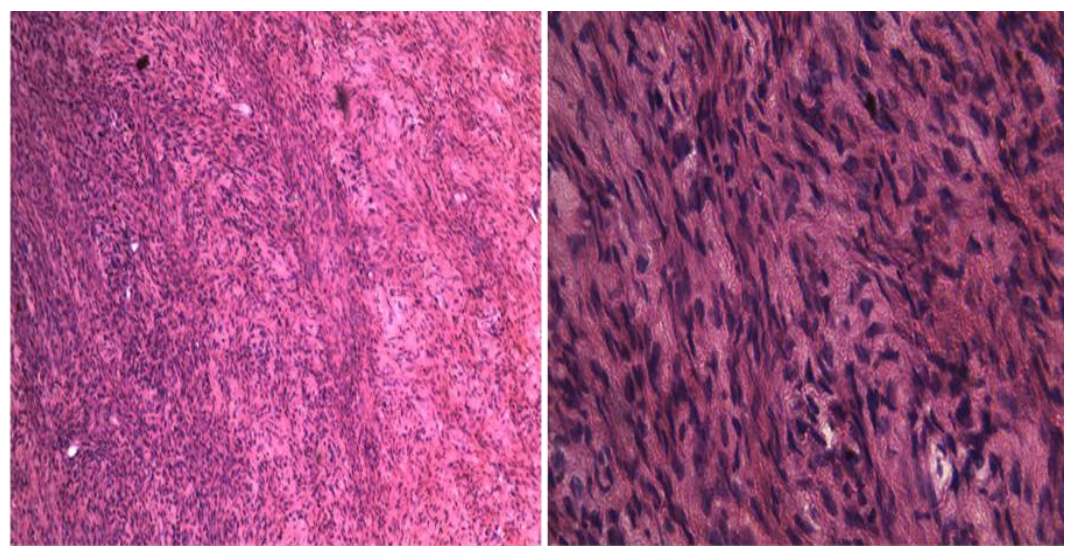

Figure $2 \& 3$ : Histological features are those of MPNST

\section{Discussion}

MPNST is defined as soft tissue tumor of putative Schwann cell lineage with anaplastic features in the form of high cellularity, cellular and nuclear pleomorphism, high mitotic rate and necrosis ${ }^{2}$.

Malignant peripheral nerve sheath tumours (MPNSTs) are rare soft tissue sarcomas of ectomesenchymal origin, comprising $5 \%$ to $10 \%$ of all soft tissue sarcomas, and often arise in anatomically discernible peripheral nerves or from neurofibromas. 50\% of MPNST $\mathrm{s}$ arises in patients with type 1 neurofibromatosis. This patient under study didn't have any stigmata of neurofibromatosis. MPNST s develops in deep seated large nerves ${ }^{3}$.

Incidence is $4.6 \%$ with neurofibromatosis and $.001 \%$ in general population. Patients with neurofibromatosis develop sarcomas usually after a latent period of ten to twenty years. The mean age of patients with sporadic MPNST is 35.3 years and mean age of MPNST with neurofibromatosis is 28.7 years ${ }^{4}$.

MPNST are large tumours, usually more than $5 \mathrm{~cm}$ in diameter. The tumour grossly appears as a large fusiform mass arising from a major nerve. Cut surface of tumour is solid, fleshy, and tan to whitish with focal areas of hemorrhage and necrosis ${ }^{1}$.

MPNST surgery is the treatment of choice. Wider margins should be obtained to limit locoregional failure, which eventually may reach the spinal cord. Chemotherapy is insensitive. Prognosis of patient with malignant MPNST is poor ${ }^{5}$. Five year survival is $54 \%$ in patients with tumours less than $5 \mathrm{cms}$ in size as compared to large tumours, which have a survival of $16 \%{ }^{4}$.

\section{Conclusion}

Nerve sheath tumours have varied clinical presentations, and a systematic approach, with judicious use of imaging, histopathology needed for diagnosis.

\section{References}

[1]. Rekha Arcot, Kavitha Ramakrishnan, Shalinee Rao: Peripheral and Cranial Nerve Sheath Tumours-A Clinical Spectrum Indian J Surg(September-October 2012)74(5):371-375

[2]. Hemalatha AL, TM Karthikeyan, Shankaranand S Bharatnur, Anil Kumar S;Malignant peripheral nerve sheath tumor in oral cavityrare site Indian J Patho Microbioi 2006;49(3):397-399

[3]. Kayo Suzuki, Taketoshi Yasuda, Takeshi Hori, Kenta Wantanabe, Masahiko Kanamori; An intraosseous malignant peripheral nerve sheath tumour of the lumbar spine without neurofibromatosis: case report and review of the literature: http://dx.doi.org/10.3892\%2Fol.2014.1987 [Pub Med] [Cross Ref] 
Retroperitoneal Malignant Peripheral Nerve Sheath Tumour without Neurofibromatosis: A Case..

[4]. S Radha, Tameem Afroz, G Satyanarayana: Paratesticular malignant peripheral nerve sheath tumour: a case report Indian J Patho Microbioi 2006; 49(4):614-615

[5]. John L. Cameron, Andrew M. Cameron; Current surgical therapy: 709-710.

[6]. Wani AA, Laherwal M, Ramzan AU, Malik NK, Lone I, Nizami FA; Malignant peripheral nerve sheath tumour of fourth ventricle and 7-8 cranial nerve complex: case report Indian Journal of Cancer :oct-dec 2015;52(4):578-579

[7]. Anghileri M, Miceli R, and Fiore M, et al. Malignant peripheral nerve sheath tumors: prognostic factors and survival in a series of patients treated at a single institution. Cancer. 2006; 107:1065-1074. [PubMed] 